\title{
Three approaches to glucose monitoring in non-insulin treated diabetes: a pragmatic randomized clinical trial protocol
}

Laura A. Young ${ }^{1,2^{*}}$, John B. Buse ${ }^{1,2}$, Mark A. Weaver ${ }^{3}$, Maihan B. Vu ${ }^{5}$, April Reese ${ }^{6}$, C. Madeline Mitchell ${ }^{2}$, Tamara Blakeney ${ }^{2}$, Kimberlea Grimm², Jennifer Rees ${ }^{2}$ and Katrina E. Donahue ${ }^{2,4}$

\begin{abstract}
Background: For the nearly 75\% of patients living with type 2 diabetes (T2DM) that do not use insulin, decisions regarding self-monitoring of blood glucose (SMBG) can be especially problematic. While in theory SMBG holds great promise for sparking favorable behavior change, it is a resource intensive activity without firmly established patient benefits. This study describes our study protocol to assess the impact of three different SMBG testing approaches on patient-centered outcomes in patients with non-insulin treated T2DM within a community-based, clinic setting.
\end{abstract}

Methods/Design: Using stakeholder engagement approach, we developed and implemented a pragmatic trial of patient with non-insulin treated T2DM patients from five primary care practices randomized to one of three SMBG regimens: 1) no testing; 2 ) once daily testing with standard feedback consisting of glucose values being immediately reported to the patient through the glucose meter; and 3) once daily testing with enhanced patient feedback consisting of glucose values being immediately reported to the patient PLUS automated, tailored feedback messaging delivered to the patient through the glucose meter following each testing. Main outcomes assessed at 52 weeks include quality of life and glycemic control.

Discussion: This pragmatic trial seeks to better understand the value of SMBG in non-insulin treated patients with T2DM. This paper outlines the protocol used to implement this study in fifteen community-based primary care practices and highlights the impact of stakeholder involvement from the earliest stages of project conception and implementation. Plans for stakeholder involvement for result dissemination are also discussed.

Trial registration: ClinicalTrials.gov NCT02033499, January 9, 2014.

Keywords: Type 2 diabetes, SMBG, Self-monitoring of blood glucose, Glycemic control, Pragmatic clinical trial

\section{Background}

Over the past decade the value of routine daily selfmonitoring of blood glucose (SMBG) in patients with type 2 diabetes (T2DM) not treated with insulin has been contentiously debated [1-8]. Proponents postulate that testing promotes better awareness of glucose levels, leading to

\footnotetext{
* Correspondence: laura_young@med.unc.edu

'Division of Endocrinology, Department of General Internal Medicine, School of Medicine, University of North Carolina at Chapel Hill, 8025 Burnett Womack Building, Campus Box \# 7172 UNC-CH, Chapel Hill, NC 27599-7170, USA

${ }^{2}$ Cecil G. Sheps Center for Health Services Research, University of North Carolina at Chapel Hill, Chapel Hill, NC, USA

Full list of author information is available at the end of the article
}

improvements in diet and lifestyle. When test results are shared with health care providers, it is argued, there is also the potential for more timely treatment modification. Competing arguments point to the costs of SMBG, both in terms of supplies (test strips and meters) and time, as well as discomfort and potentially quality of life. As a result no clear consensus exists regarding SMBG monitoring in non-insulin treated patients with T2DM.

In an attempt to make SMBG more convenient and patient-centric, much effort has been placed in improving SMBG technology [9]. Early studies that marry technology and SMBG been mixed, with some showing a benefit on glycemic control and others showing no benefit $[1,10]$. 
Few trials have pragmatically assessed the utility of SMBG monitoring in real-life settings, like the busy primary care practice where the majority of non-insulin treated patients with T2DM are managed. With patients taking a more directive role in their health care, a large focus is being placed on how usable these glucose-monitoring tools are in the real world. Few studies have focused on the health care provider side of this issue. In fact, studies of 'enhanced' SMBG, where both the patient and the provider were engaged in SMBG interpretation, found A1c reductions along the magnitude of $0.5 \%$ [4, 11-13]. As additional 'enhanced' intervention SMBG studies have been added to the literature $[13,14]$, more recent reviews and meta-analyses have drawn conclusions more in favor or testing $[4,15]$. This pattern suggests that, for SMBG to be an effective self-management tool in non-insulin treated T2DM, the patient and the health care provider must both actively engage in performing, interpreting, and acting upon the SMBG values.

Given these unanswered questions regarding the impact of SMBG on patient quality of life and other patient reported outcomes, there is a growing interest by patients and other stakeholders who are looking for data that will help them make better, informed decisions about their self-care when no standard of care exists and providers make recommendations based upon their experience and preferences, like SMBG monitoring in T2DM. Our overarching goal is to answer the following questions: Is SMBG testing effective for people with non-insulin treated T2DM in terms of either A1c or quality of life (QOL)? We will also examine outcomes from SMBG in patients with different baseline characteristics. Our primary outcomes include change in glycemic control over 52 weeks and change in QOL over 52 weeks. The purpose of this paper is to describe our study methods and the unique aspects of stakeholder engagement that have been employed during the design and implementation of the study.

\section{Methods/Design}

\section{Study overview}

The overarching goal of this proposal is to assess the impact of three different SMBG testing approaches on patient-centered outcomes in patients with non-insulin treated T2DM within the real-world, clinic setting. In this pragmatic trial, 450 patients will be randomized to one of the following three SMBG testing regimens: 1) no SMBG testing, 2) once daily SMBG testing with standard patient feedback consisting of glucose values being immediately reported to the patient through the glucose meter, and 3) once daily SMBG testing with enhanced patient feedback consisting of glucose values being immediately reported to the patient PLUS automated, tailored feedback messaging following each SMBG testing event delivered to the patient through the glucose meter.

\section{Recruitment}

We will recruit 450 patients from primary care practices within the central North Carolina area. Patients will be randomized to one of the three study arms: 1) no SMBG testing; 2) once daily SMBG testing with standard patient feedback which includes the current glucose value; and 3) once daily SMBG testing with enhanced patient feedback which includes the current glucose value and a personalized message about the current glucose value. We have elected to use an FDA approved, cellularly-enabled glucometer device given its capabilities to deliver messaging to patients in real time. Participants in both the standard feedback and enhanced feedback groups will all receive the glucometer. Patients will be followed for 1 year. The first two study groups represent SMBG testing approaches commonly utilized in clinical practice, while the third incorporates cutting edge glucose monitoring tools now on the market. During routine clinic visits, health care providers will be guided to modify therapies based upon American Diabetes Association (ADA) guidelines, which focus on A1c values and SMBG values if available. Patients in groups 2 and 3 will also receive training in obtaining and interpreting SMBG values. SMBG values will be systematically evaluated at routine clinic visits.

\section{Stakeholder Engagement}

As part of this grant, stakeholders were encouraged to play a role in providing perspective to the research questions and methodologies chosen, and patient related outcomes. There were several stakeholders that provided a critical role in providing care advocacy and education for persons with diabetes in North Carolina, which included patient groups community members at risk individuals policy makers providers industry and professional organizations. Given all of these stakeholder groups involved in the area of diabetes we chose a broad yet appropriately sized swath of this community. This included the North Carolina Diabetes Advisory Council, a patient advisory board, a community advisory board, health care providers, American diabetes Association representative, National Diabetes Education Program representation, as well as patients involved in the Diabetes registry and Glucometer manufacturers. Two primary methods were planned for continuing collaboration with these stakeholder groups. This included attending regularly scheduled meetings the larger organizations on a pre-stipulated basis and holding key stakeholder teleconferences with a small group $(\sim 10)$ of representative stakeholders. 


\section{Patient identification and recruitment}

A multi-pronged approach will be utilized to identify study participants. First, patients who have non-insulin treated T2DM will be identified using the Carolina Data Warehouse (CDW) or by chart review in the participating practice. Patients will receive an invitation letter by mail, signed by their health care provider describing the study. Any patient not interested in participating will be able to opt out of further contact by returning a post card or calling a toll-free number. If no opt out call is made within 1 week of sending the letter, telephone contact will be attempted on up to three different occasions at three different times of day. Second, we will place recruitment flyers within the participating practices that will be visible in waiting areas and exam rooms. Clinic staff will be encouraged to discuss the study with potentially eligible patients during routine clinic activities (i.e. while collecting vital signs and during check-in or check-out). The UNC field coordinators will communicate regularly with practice staff regularly regarding potentially eligible

All potentially eligible patients will be screened for eligibility by phone call. Overall inclusion and exclusion criteria are listed in Table 1 . The call will take about 15 min, during which a member of the study team describes the study, answer questions, and conducts a short set of simple screening questions. Eligible interested patients complete an assessment visit with a research coordinator. To decrease patient burden and further engage participating practices, assessment visits occur at the patient's primary care office. Assessments are separate from their appointment with their primary care provider, and may or may not occur on the same day as a regularly scheduled clinic visit, though for patient convenience we make every effort to coordinate the assessments with a regular clinic visits. During these assessments, the research coordinator reviews the study details in greater depth, verify all inclusion and exclusion criteria, and obtain written informed consent.

Table 1 Monitor trial inclusion and exclusion criteria

\begin{tabular}{|c|c|}
\hline Inclusion Criteria & Exclusion Criteria \\
\hline $\begin{array}{l}\text { T2DM diagnosis } \\
\text { Age } \geq 30\end{array}$ & $\begin{array}{l}\text { Sees an endocrinologist or } \\
\text { other diabetes specialist }\end{array}$ \\
\hline $\begin{array}{l}\text { An established patient at the } \\
\text { participating practice }\end{array}$ & Use of insulin. \\
\hline$\geq 6.5 \%$ but $\leq 9.5 \%$ & Pregnancy \\
\hline $\begin{array}{l}\text { Willing to comply with random } \\
\text { assignment into a study group }\end{array}$ & $\begin{array}{l}\text { Plans to relocate in the next } 12 \\
\text { months. }\end{array}$ \\
\hline $\begin{array}{l}\text { No history of significant issues with } \\
\text { hypoglycemia }\end{array}$ & $\begin{array}{l}\text { Has other conditions (e.g. renal or } \\
\text { cardiovascular disease), factors } \\
\text { (e.g. frailty) or comorbidities } \\
\text { (e.g. cancer) that might put the } \\
\text { patient at risk }\end{array}$ \\
\hline
\end{tabular}

\section{Data collection \\ Baseline}

Eligible patients interested in enrolling for the study are subsequently scheduled for the initial visit with the study Field Coordinator. During this initial visit, the Field Coordinator reviews and obtains written informed consent from the patient. A signed copy is provided to the participant. After written informed consent has been received, blood samples are obtained along with height and weight. Standardized surveys are completed, and patients have the option of completing them independently or with the assistance of the Field Coordinator.

\section{Randomization}

After providing informed consent, baseline A1c, and completing the baseline study interview, participants will be randomized to one of the three treatment arms using sequentially numbered, opaque, sealed envelopes. The allocation sequence is generated using computergenerated randomly permuted blocks of random sizes. The randomization is stratified by study practice. The Field Coordinator reviews the treatment assignment with the patient, using a standardized script, provide the initial training and supplies necessary for participation in the study and answer any remaining questions (Fig. 1).

\section{2 week follow-up}

Patient will have blood drawn for a A1c. Patient participants will complete a follow-up interview that includes demographic, health history, and quality of life questions. Weight measurement will be collected.

\section{Measures}

The two primary outcomes of the study are change in A1c and change in health related quality of life (HRQOL). The A1c was chosen due to its use in prior studies and its standing as a measure on which: 1) providers evaluate patients; 2) payers evaluate providers; and 3) patients evaluate themselves and their providers [2]. Though HRQOL is also critically important to patients, few studies have rigorously examined the impact of SMBG on HRQOL. Based on these facts and the recommendations of our stakeholder groups, HRQOL is a co-primary outcome for this pragmatic trial. We will utilize the Short Form 36 (SF-36) to assess overall quality of life. We will use the physical component score (PCS) and the mental component score (MCS), with scores standardized to a normal distribution (mean $=50$ and standard deviation[SD] = 10) [16]. It has been widely used and validated in medical studies generally and diabetes studies in particular [17-20]. All primary and secondary outcomes are outlined in Table 2 . Because the 52-week time point may not align with a scheduled 


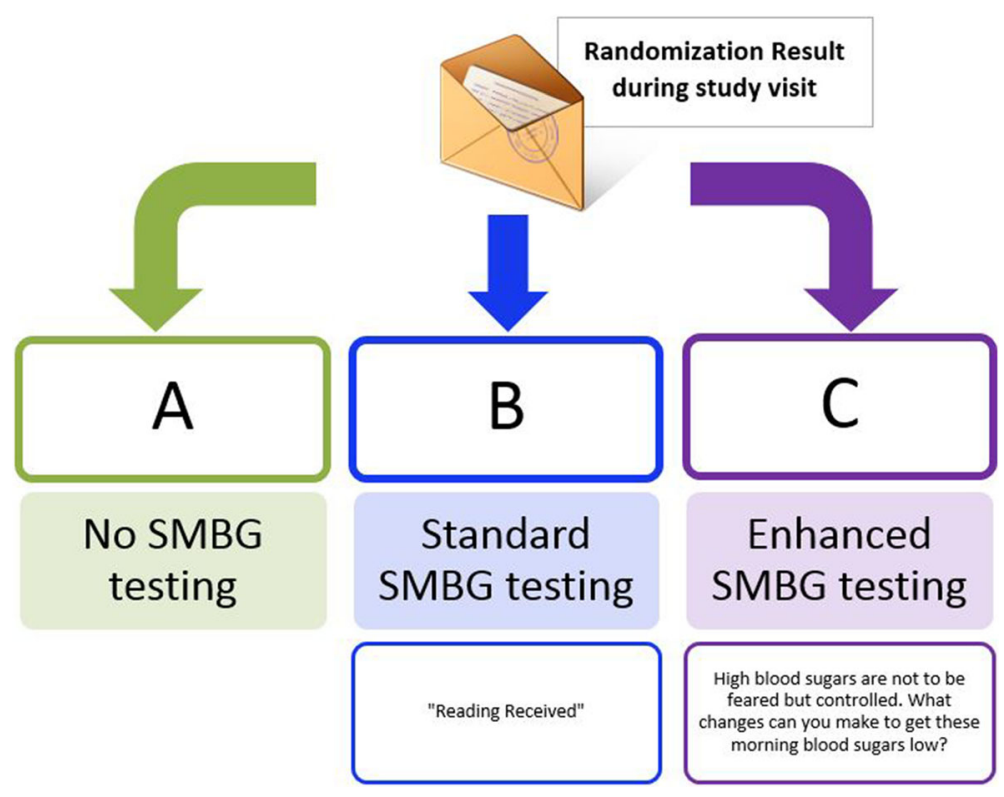

Fig. 1 Study Arms

primary care visit, we have defined this time point as 52 \pm 6 weeks from the baseline visit.

The following secondary endpoints will be collected from patient participants at baseline and 52-weeks. 1) Problem Areas in Diabetes (PAID) to assess psychological and social stress associated with diabetes [21-23], 2) Diabetes Symptom Checklist (DSC) to measure of diabetes-related symptom frequency and perceived severity during the prior month covering six symptom categories: hyperglycemic, hypoglycemic, cardiac, neuropathic, psychological, and vision-related [24-26] 3) Summary of Diabetes Self Care Activities (SDCA) survey to measure self-management activities (diet, exercise, blood glucose testing, foot-care, and smoking status and a supplemental medication adherence question [27], 4) the Diabetes Treatment Satisfaction Questionnaire (DTSQs) standard version to assess this diabetes treatment satisfaction [28], 5) Diabetes-Specific Self-Efficacy using the Diabetes Empowerment Scale Short Form $[29], 6)$ Patient-Provider Communication using the Communication Assessment Tool [30].

\section{Hypoglycemia Frequency}

At the 52-week follow-up visit, participants are asked to report the number of hypoglycemic episodes that required intervention by a caregiver or by medical or paramedical personnel since enrolling in the study. For each episode, the participant will be asked whether EMS was called or led to an urgent care clinic or emergency department visit, or hospitalization. Each report is reviewed by the study principal investigators and coinvestigator to determine whether the event is study related. A summary report is submitted to the UNC Institutional Review Board.

\section{Health Care Utilization}

Utilization outcomes will include inpatient, outpatient and emergency department utilization during the 52week study period. UNC visits will be collected through the EHR while non UNC visits will be captured at the 52 week study visit via patient interview with the study coordinator.

Table 2 Primary and secondary patient-centered outcomes

\begin{tabular}{lllll}
\hline & Clinical Outcomes & Time & Patient Reported Outcomes & Time \\
\hline Primary & Glycemic control (A1c) & Baseline/52 weeks & Quality of life (QOL) & Baseline/52 weeks \\
Secondary & Hypoglycemia frequency & Baseline/52 weeks & DM-related QOL & Baseline/52 weeks \\
& Health care utilization & Baseline/52 weeks & DM self-care & Baseline/52 weeks \\
& Treatment regimen modification & Baseline/52 weeks & DM treatment satisfaction & Baseline/52 weeks \\
& & DM-related self-efficacy & Baseline/52 weeks \\
& & Patient-provider communication & Baseline/52 weeks \\
& & &
\end{tabular}




\section{Adverse Events}

Any reported adverse events will be categorized as related or not related to the study intervention by the investigators. These events will be reported in real time to the principal investigators for confirmation and review. Regardless of causality, all unanticipated Serious Adverse Events will be reported to the IRB for review.

\section{Qualitative Assessment}

To gain a deeper understanding of patients' and health care providers' experiences with each of the SMBG testing approaches, including facilitators and barriers to dissemination, we will conduct focus group discussions at the UNCPN practices.

Qualitative Assessment of Patient Outcomes Individual phone interviews will be conducted by researchers skilled in qualitative data collection. At each participating practice, we will interview approximately 6 patients who have completed the study. Two patient participants from each arm will be recruited for each of the 13 practices ( $N=78$ patients). Every effort will be made to recruit a range of high and low engagement for testing arms. Interviews will last approximately 30-45 min and will include these topics: impressions of and experience with SMBG prior to and during the trial, including using a wireless glucometer and messaging system, and impressions of using the downloaded SMBG reports at clinic visits, interactions with providers about diabetes care before and during the study, and strategies for dissemination and communication. All participants will review, sign, and be given a copy of the IRB-approved informed consent document specific to the focus groups.

Qualitative Assessment of Health Care Provider Outcomes Focus groups (one per practice) with 5-10 physicians and nursing staff will also be conducted. by researchers skilled in qualitative data collection. Focus groups will be conducted once at least $80 \%$ of that practice's enrolled study participants will have completed their 52-week follow-up visit. Key discussion topics will include: impressions regarding the usefulness of SMBG summary reports and accompanying treatment algorithm; experience with recommending SMBG for patients with NIT DM prior to and during the trial (and for both enrolled and non-enrolled patients); and perceived benefits/problems arising from being a practice site for this study. All participants will review and sign IRB-approved informed consent documents prior to participation. We will also collect age, gender, race/ethnicity, full-time/part-time employment status, years in practice, and area of practice.

\section{Sample Size}

We desire high power for our primary between-arm comparison as well as reasonable power to detect an important effect modifier, should one exist. In a recent Cochrane Review, the estimated mean 12-month differences between SMBG and control groups were-0.13 and $-0.52 \%$, respectively, for patients diagnosed more than and less than 1 year prior to the study [1]. Assuming approximately equal enrollment of newly diagnosed and long term patients, this implies a mean difference of $-0.325 \%$. Based on results from one of the larger and better quality trials conducted to date, we assume the standard deviation for $\Delta \mathrm{A} 1 \mathrm{c}$ would be $0.8 \%$ [4]. We assume no more than $10 \%$ loss to follow-up. Given these assumptions, randomizing 150 patients per group would provide at least $90 \%$ power for the primary comparison for A1c at the 0.05 significance level. This same sample size would provide at least $80 \%$ power for the primary comparison if the true standard deviation were as high as $1.0 \%$. For comparing mean change in HRQOL between groups, we will use the physical and mental component scores of the SF-36, both of which range from 0 to 100. Because we are interested in two different aspects of QOL, we will apply a Bonferroni correction and assess each comparison at the 0.025 level. Based on observed results from the ZODIAC-17 SMBG study, we conservatively assume that the standard deviation for the change scores for either component will be 10 points [2]. Under these assumptions, randomizing 150 patients per group with no more than $10 \%$ loss will provide at least $80 \%$ power to detect an overall difference between groups if the mean difference between the highest and lowest groups is at least 4 points.

\section{Data Management and Statistical Analysis Plan}

All data will be systematically coded and double-entered into a data system stored on a secure server according to institutional, state, and federal policies. Contact information was obtained from electronic health records and stored on a secure, password-protected server with access limited to study staff and will be destroyed after 5 years as required.

A detailed statistical analysis plan, finalized prior to enrolling the first patient, is available from the authors upon request; here, we provide key elements of that plan for analysing the co-primary outcomes. The biostatistician and principal investigators are blinded to treatment assignments, but the study field staff are not blinded. For primary analyses, all randomized patients will be analysed according to their randomized group regardless of the extent to which they performed SMBG (intentionto-treat). Missing 52-week outcome data will be ignored for the primary analyses. First, we will compare change in A1c from baseline through 52 weeks across the three 
randomization groups using an analysis of covariance (ANCOVA) conducted at the 0.05 significance level. This ANCOVA model will control for baseline A1c, prior use of SMBG, duration of T2DM, baseline antihyperglycemic treatment, age, race/ethnicity, health literacy, and number of baseline comorbidities. If the overall null hypothesis of no difference between the three groups is rejected, we will compare each SMBG group to the no testing group separately using the DunnettTamhane Step-Up procedure for multiple comparisons to control the family-wise error rate at 0.05 (69). We will also conduct a contrast test comparing the average of the two SMBG groups to the no testing group at the 0.05 level. Similar ANCOVA models will compare change in SF-36 physical and mental component scores between groups, controlling for the same baseline variables as for A1c. Because we are examining two aspects of HRQOL, we will apply a Bonferroni correction and assess each comparison at the 0.025 level. We will not adjust for multiple comparisons due to the co-primary endpoints of A1c and HRQOL. In addition, we will assess for potential effect modification for each of the baseline variables included in the models by adding appropriate interaction terms to the ANCOVA model one at a time. Each interaction term will be tested separately at the 0.05 significance level. Only if the associated interaction term is significant, similar contrasts to those described above will be assessed within the relevant subgroups. Similar ANCOVA methods will be used to compare groups on each of the secondary outcomes. These tests will each be conducted at the 0.05 significance level with no adjustments for multiple comparisons.

\section{Baseline Statistics}

Table 3 highlights the baseline statistics of the 450 subjects that have been recruited into the study.

\section{Discussion}

\section{Engaging Stakeholders}

We designed the Monitor Trial using a pragmatic design that was informed by our stakeholders. Increasingly organizations like the Patient Centered Outcome Research Institute (PCORI) are encouraging the involvement of stakeholders in all aspects of research from study inception, design, implementation, analysis, and dissemination of results. Although the benefits and/or pitfalls of including stakeholders broadly has yet to be fully understood, this approach when executed effectively holds great promise for helping patients and providers make better, more informed choices about their health and healthcare. Below we describe how we have engaged our stakeholders to date and our plans for continued engagement.
The study team engaged a multitude of stakeholders during the inception of the study to ensure that issues and outcomes that mattered most to patients and heath care providers were fully considered. We engaged patients primarily via two avenues. First we attended two Patient Advisory Board (PAB) meetings to interact in real-time with patients, care givers and community members affected by diabetes. We also electronically surveyed patients from the UNC Diabetes Care Center Patient Registry, which at the time of the survey include over 2000 patients with diabetes across North Carolina. From these interactions we learned that there is a perceived direct link between SMBG and quality of life. Additionally the feedback we received confirmed our beliefs that quality of life is important to patients. Registry respondents reported that they would use SMBG more frequently if it held the potential to positively impact quality of life. During discussions with the PABs the topic of patient-provider communication arose. Insights from patients and caregivers highlighted the fact that patient-provider communication is an important issue and that avenues to improve this should be developed. It was viewed that consistently reviewing SMBG results together is an opportunity to build partnerships between patients and their health care providers. Registry respondents underlined current inconsistencies related to how SMBG values are utilized during clinic visits. Most (59\%) reported that they believed it was important for medical providers to review SMBG values, but only $38 \%$ reported their health care providers always reviewed SMBG logs and provided feedback. Based upon this feedback we solidified the study team's initial premise that for SMBG to be effective, both the patient and provider must be engaged in the process and as a result designed the study to include treatment recommendations that would be generated for each health care provider at the time of the clinic visit. These treatment recommendations are intended to serve as a guide to inspire discussion between patients and health care providers. To objectively capture this, we included a measure of patient-provider communication that is completed at baseline and end of study. We will be collecting qualitative data related to this topic at the conclusion of the study as well to provide greater insight.

Input from health care providers was also utilized during the design of this project. Early on the study team engaged the UNCPN membership during a large network-wide meeting. We discussed with the providers the varied recommendations made to patients related to SMBG monitoring in the real-world setting. Providers reported that the believed the primary question of SMBG monitoring effectiveness in T2DM to be an unsettled, high priority topic in primary care. This message was underscored by findings from the registry. Of the 62 
Table 3 Baseline interview

\begin{tabular}{|c|c|c|c|c|c|}
\hline & Mean (sd) & Min & Max & N (\%) & \# Missing \\
\hline \multicolumn{6}{|l|}{ Demographics } \\
\hline Age (years) & $60.5(11.5)$ & 30.8 & 91.8 & & \\
\hline Male & & & & $207(46.0)$ & \\
\hline \multicolumn{6}{|l|}{ Race } \\
\hline American Indian or Alaskan Native & & & & $3(0.7)$ & \\
\hline Asian & & & & $9(2.0)$ & \\
\hline Black or African-American & & & & $148(33.0)$ & \\
\hline $\begin{array}{l}\text { Native Hawaiian/ } \\
\text { Pacific Islander }\end{array}$ & & & & $1(0.2)$ & \\
\hline White & & & & $279(62.3)$ & \\
\hline Other (African, Filipino, Hispanic) & & & & $3(0.7)$ & \\
\hline Multiracial (1 = white + other, Indian) & & & & $5(1.1)$ & \\
\hline Refused (e.g., human race) & & & & & 2 \\
\hline \multicolumn{6}{|l|}{ Ethnicity } \\
\hline Latino/Hispanic & & & & $8(1.8)$ & 1 \\
\hline \multicolumn{6}{|l|}{ Education } \\
\hline Less than high school graduate & & & & $25(5.6)$ & \\
\hline High school graduate or GED & & & & $107(23.8)$ & \\
\hline Some college or associate's degree & & & & $164(36.5)$ & \\
\hline 4-year college degree & & & & $92(20.5)$ & \\
\hline Graduate degree & & & & $61(13.6)$ & \\
\hline Refused & & & & & 1 \\
\hline \multicolumn{6}{|l|}{ Marital status } \\
\hline Married & & & & $292(64.9)$ & \\
\hline Widowed & & & & $37(8.2)$ & \\
\hline Living together & & & & $4(0.9)$ & \\
\hline Single & & & & $64(14.2)$ & \\
\hline Separated & & & & $7(1.6)$ & \\
\hline Divorced & & & & $46(10.2)$ & \\
\hline \multicolumn{6}{|l|}{ Comorbidities } \\
\hline \# comorbidities & $3.4(1.8)$ & 0 & 10 & & \\
\hline Heart disease & & & & $86(19.2)$ & 1 \\
\hline High blood pressure & & & & $340(75.6)$ & \\
\hline Lung disease & & & & $30(6.7)$ & \\
\hline Stroke & & & & $29(6.4)$ & \\
\hline High Cholesterol & & & & $299(67.0)$ & 4 \\
\hline Kidney disease & & & & $32(7.1)$ & \\
\hline Liver disease & & & & $18(4.0)$ & \\
\hline Anemia or other blood disease & & & & $61(13.6)$ & \\
\hline Cancer & & & & $76(16.9)$ & \\
\hline Depression/anxiety & & & & 139 (30.9) & \\
\hline Arthritis & & & & $210(46.8)$ & 1 \\
\hline Chronic back pain & & & & $112(24.9)$ & \\
\hline Autoimmune disease & & & & $14(3.1)$ & \\
\hline
\end{tabular}


Table 3 Baseline interview (Continued)

\begin{tabular}{|c|c|c|c|c|}
\hline Stomach or bowel disease & & & & $83(18.4)$ \\
\hline \multicolumn{5}{|l|}{ Healthcare utilization } \\
\hline Have you seen your primary care provider? & $3.7(2.4)$ & 1 & 30 & \\
\hline Have you gone to an urgent care clinic? & $0.5(1.1)$ & 0 & 12 & \\
\hline Have you been seen in the Emergency Room & $0.4(1.1)$ & 0 & 12 & \\
\hline Have you been hospitalized overnight? & $0.2(0.5)$ & 0 & 6 & \\
\hline Have you had someone call EMS for you? & $0.1(0.4)$ & 0 & 5 & \\
\hline \multicolumn{5}{|l|}{ DM-related } \\
\hline Nephropathy or other diabetes-related kidney disease & & & & $11(2.4)$ \\
\hline Diabetic retinopathy or other diabetes-related eye disease & & & & $20(4.4)$ \\
\hline Neuropathy or other diabetes-related nerve damage & & & & $65(14.4)$ \\
\hline Years with T2DM diagnosis & $8.2(7.6)$ & 0.1 & 50.0 & \\
\hline \# Testing $=$ Yes & & & & $338(75.1)$ \\
\hline If yes, how often: at least daily & & & & $129(38.2)$ \\
\hline If yes, how often: not daily, but > 1X/week & & & & $117(34.6)$ \\
\hline If yes, how often: a few times a month & & & & $50(14.8)$ \\
\hline If yes, how often: once a month & & & & $21(6.2)$ \\
\hline If yes, how often: less than once a month & & & & $21(6.2)$ \\
\hline If yes, how often told: at least daily & & & & $187(55.5)$ \\
\hline If yes, how often told: not daily, but > 1X/week & & & & $53(15.7)$ \\
\hline If yes, how often told: a few times a month & & & & $14(4.2)$ \\
\hline If yes, how often told: once a month & & & & $2(0.6)$ \\
\hline If yes, how often told: < once a month & & & & $1(1.2)$ \\
\hline If yes, how often didn't know & & & & $77(22.9)$ \\
\hline If Testing $=$ No, ever tested? & & & & 78 (69.6) \\
\hline If yes, at least daily & & & & $55(70.5)$ \\
\hline If yes, not daily, but > 1X/week & & & & $10(12.8)$ \\
\hline If yes, a few times a month & & & & $3(3.9)$ \\
\hline If yes, once a month & & & & $1(1.3)$ \\
\hline If yes, less than once a month & & & & $9(11.5)$ \\
\hline If yes, how long did you test: $<=1$ month & & & & $8(10.3)$ \\
\hline If yes, how long did you test: $<1$ year $>1 \mathrm{X} /$ month & & & & $35(44.9)$ \\
\hline If yes, how long did you test: 1 year & & & & $12(15.4)$ \\
\hline If yes, how long did you test: $>1$ year $<5$ years & & & & $17(21.8)$ \\
\hline If yes, how long did you test: $>=5$ years & & & & $6(7.7)$ \\
\hline$\#$ Testing = No, why not? & & & & $112(24.9)$ \\
\hline Not told to test & & & & $50(44.6)$ \\
\hline Costs & & & & $18(16.1)$ \\
\hline Pain & & & & $8(7.1)$ \\
\hline Disruptive & & & & $4(3.6)$ \\
\hline Does not understand why should test & & & & $7(6.25)$ \\
\hline Constant reminder of DM & & & & $10(8.9)$ \\
\hline Other reason & & & & $30(26.8)$ \\
\hline
\end{tabular}


Table 3 Baseline interview (Continued)

\begin{tabular}{|c|c|c|c|c|c|}
\hline \multicolumn{6}{|l|}{$\overline{\text { Medications and BMI }}$} \\
\hline \# of prescription medications & $6.2(3.4)$ & 0 & 17 & & \\
\hline \# of DM medications & $1.4(0.9)$ & 0 & 5 & & \\
\hline BMI (max weighs 523 lb) & $34.3(7.7)$ & 20.8 & 75.0 & & \\
\hline \multicolumn{6}{|l|}{ Primary Endpoints } \\
\hline Hemoglobin A1c (\%) & $7.6(1.1)$ & 5.7 & 13.1 & & \\
\hline SF36-Physical Health & $47.4(8.9)$ & 10.4 & 63.0 & & \\
\hline SF36-Mental Health & $53.3(9.2)$ & 9.5 & 68.0 & & \\
\hline \multicolumn{6}{|l|}{ Secondary Endpoints ${ }^{a}$} \\
\hline Diabetes Empowerment Scale—Short Form & $4.3(0.5)$ & 1.1 & 5 & & 1 \\
\hline Diabetes Symptom Checklist—Revised & $20.4(21.4)$ & 0 & 107.2 & & \\
\hline Diabetes Treatment Satisfaction—satisfaction & $31.8(5.1)$ & 6 & 36 & & 6 \\
\hline Diabetes Treatment Satisfaction-perceived control & $2.7(2.6)$ & 6 & 12 & & 23 \\
\hline Problem Areas in Diabetes & $13.1(16.2)$ & 0 & 82.5 & & \\
\hline \multicolumn{6}{|c|}{ Summary of Diabetes Self-Care Activities (SDSCA) (\# of days in last 7) } \\
\hline General Diet & $4.3(2.2)$ & 0 & 7 & & 1 \\
\hline Special Diet & $4.1(1.8)$ & 0 & 7 & & \\
\hline Carb spacing & $4.1(2.4)$ & 0 & 7 & & \\
\hline Exercise & $2.9(2.2)$ & 0 & 7 & & \\
\hline Blood glucose testing (only \# times tested) & $2.6(2.8)$ & 0 & 7 & & 1 \\
\hline Foot care & $3.2(2.5)$ & 0 & 7 & & \\
\hline Communication Assessment Tool-MD items & $4.5(0.7)$ & 1.5 & 5 & & 1 \\
\hline Communication Assessment Tool—staff item & $4.6(0.7)$ & 1 & 5 & & \\
\hline \multicolumn{6}{|l|}{ Health Literacy } \\
\hline Newest Vital Sign & $3.8(2.0)$ & 0 & 6 & & 2 \\
\hline Newest Vital Sign ( $\%>4=$ literate) $($ median $=4)$ & & & & $235(61.8)$ & 2 \\
\hline
\end{tabular}

${ }^{\mathrm{a}} \mathrm{Min} / \mathrm{max}$ may contain a decimal if missing data within the allowable limit were present

registry respondents with T2DM not using insulin, 90\% reported that they included SMBG as part of their selfcare plan. Self-reported SMBG frequencies were: daily, $37.5 \%$; several times per week, 37.5\%; several times per month, 20\%, and less than once per month, 5\%. The other main take home point distilled from our conversations with providers related to the need for the proposed study to be "efficient" and minimally disruptive to the day-to-day clinic flow. This feedback guided the intervention development. Additional feedback during the planning phase of the MONITOR Trial was obtained by several other organizations outlined in Table 4.

We continue to engage the stakeholders during the grant process with periodic phone calls where they have provided input on that best methods of patient recruitment, dissemination opportunities, patient scripts used during the baseline interview process. The overall goal of involving various stakeholder groups is to have key people with a variety of perspectives involved in planning, oversight, analysis, and dissemination. We consider this collaboration essential for not only designing and conducting research study that is responsive to the needs and practical question non-insulin treated type 2 diabetes patient's and caregivers daily but also to disseminating our findings.

\section{Clinical Implications}

Self-monitoring of blood glucose in patients with diabetes has traditionally been considered to be a pillar of diabetes self-care. Scientific examinations of SMBG in T2DM have provided mixed results. An early epidemiological evaluation of the issue using a retrospective, longitudinal analysis showed that nonfatal micro- and macrovascular event rates along with fatal event rates were lower in individuals performing SMBG routinely as compared to those who were not [3]. A multitude of clinical trials followed. Several showed a significant benefit from SMBG testing on improving glycemic control [4-7], while others found no evidence of benefit [16]. In fact, some studies even suggested harm from routine SMBG in patients with T2DM, specifically, 
Table 4 Stakeholders and the input in the study design

\begin{tabular}{|c|c|c|}
\hline Stakeholders & Input provided & How it shaped our design \\
\hline $\begin{array}{l}\text { Diabetes Advisory Council/ } \\
\text { State Department of Health } \\
\text { (DAC) }\end{array}$ & $\begin{array}{l}\text { Consider health literacy issues of patients } \\
\text { Policy subgroup would be useful to engage } \\
\text { for this work }\end{array}$ & $\begin{array}{l}\text { - Engaged the Center for Diabetes Translation } \\
\text { and Research literacy core to join our team and } \\
\text { assist with message tailoring } \\
\text { - Tailoring algorithm that could be used in office } \\
\text { - Brought in Diabetes Advisory Council as a policy } \\
\text { subgroup }\end{array}$ \\
\hline UNC Patient Advisory Board & $\begin{array}{l}\text { Emphasize quality of life questions (e.g, Can } \\
\text { I feel better or improve my ADLs?) }\end{array}$ & Added quality of life to outcomes \\
\hline $\begin{array}{l}\text { Greensboro Community } \\
\text { Advisory Board }\end{array}$ & $\begin{array}{l}\text { Important outcomes: Quality of life, hypoglycemia, } \\
\text { health care service use, and patient empowerment. } \\
\text { CMEs for providers, } \\
\text { Query patient/provider community care }\end{array}$ & $\begin{array}{l}\text { Hypoglycemia added as an outcome } \\
\text { CME added for providers } \\
\text { Added survey questions about patient-provider } \\
\text { communication }\end{array}$ \\
\hline Diabetes Center Patient Database & A $1 C$ is important in addition to Quality of life & A $1 \mathrm{C}$ designated as a primary outcome \\
\hline UNCPN Medical Directors & $\begin{array}{l}\text { Testing is quite variable in real world clinical } \\
\text { settings }\end{array}$ & $\begin{array}{l}\text { Designed three-armed plan to address this reality } \\
\text { and better respond to pragmatic patient issues. }\end{array}$ \\
\hline
\end{tabular}

higher rates of depression and increased cost without accompanying benefits [31].

Given these mixed results, a series of meta-analyses and systematic reviews were conducted to investigate the benefit or lack thereof of SMBG on glycemic lowering in patients with T2DM $[1,9,11-14]$. While meta-analyses can be a useful way to assess the clinical effectiveness of an intervention, they are limited by the quality and comparability of the clinical trials included in the analyses. Issues of sample size, duration/details of the intervention, and patient characteristics (e.g., newly diagnosed vs. longer duration of disease; baseline A1c level), varied considerably across the available studies. Given these critical differences, it is perhaps not surprising that the results of the meta-analyses have also shown conflicting results. However, the overall conclusion has been that SMBG is likely not cost effective for this population of patients $[1,9,12]$.

Perhaps most important to understanding these mixed results is the fact that the question being addressed by the studies is itself not consistent, falling generally into two camps: 'simple' SMBG and 'enhanced' SMBG. In studies testing simple SMBG, patients conducting SMBG were compared to patients who were not. In evaluations of 'enhanced' SMBG, intervention group patients and/or providers were given education or feedback such that they were better able to interpret SMBG results and use them in a meaningful way with regard to lifestyle changes and treatment modification. Among tests of 'simple' SMBG, A1c levels were reduced on average by $0.2 \%$, an amount that was statistically significant in these studies, but of doubtful clinical significance [12, 13]. Studies of 'enhanced' SMBG found A1c reductions closer to $0.5 \%[7,12,15,16]$. As additional 'enhanced' intervention SMBG studies were added to the literature $[13,14]$, more recent reviews and meta-analyses have drawn conclusions more in favor or testing [4, 15]. This pattern suggests that, for SMBG to be an effective selfmanagement tool in T2DM, the patient and the health care provider must both actively engage in performing, interpreting, and acting upon the SMBG values.

In an attempt to make SMBG more convenient and patient-centric, much effort has been place in improving the technology supporting SMBG. Like their nontechnological predecessors the results from these early studies that marry technology and SMBG in an effort to make the process more convenient have been mixed, with some showing a benefit on glycemic control and others showing no benefit. With patients' taking a more directive role in their health care, a large focus is being place on how usable these glucose-monitoring tools are in the real world. Generally speaking many of the applications need to focus on improved usability, perceived usefulness, and sustained adoption of the technologies [6]. Web-based applications, applications with easy-touse mechanisms, and applications without location restrictions (can be used at home) have been most useful [7]. Furthermore, few studies have focused on the health care provider side of this issue. Allowing consistent, hassle free access to SMBG values for providers is an equally important part of the equation. In fact, studies of 'enhanced' SMBG, where both the patient and the provider were engaged in SMBG interpretation, found A1c reductions along the magnitude of $0.5 \%[7,12,15,16]$. As additional 'enhanced' intervention SMBG studies have been added to the literature [13, 14], more recent reviews and meta-analyses have drawn conclusions more in favor or testing $[4,15]$. This pattern suggests that, for SMBG to be an effective self-management tool in noninsulin treated T2DM, the patient and the health care provider must both actively engage in performing, interpreting, and acting upon the SMBG values. 
One issue often neglected in prior work is the fact that SMBG could impact patient quality of life (QOL) both positively and or negatively. Testing itself is a burden and could act as a constant reminder of one's less than ideal health status [32]. On the other hand, testing may provide a sense of agency, improving a patients self of self-efficacy and hope for maximizing health and independence into the future [32]. A recent Cochrane Review identified only a handful of studies that had examined health-related quality of life (HRQOL), wellbeing, or patient satisfaction [1]. While these studies did not find clinically relevant differences in HRQOL for those who do or do not test, the review indicated future research was needed [1]. In one study, HRQOL initially decreased, but follow-up qualitative interviews showed that patients in the testing groups experienced an increased awareness of illness [4]. While both simple and 'enhanced' versions of SMBG were evaluated, the enhanced version included only training in the meaning of the results and encouragement to explore how lifestyle and dietary choices affect test values). Without more hands-on use of results (e.g., reports provided to the care provider), patients might have felt more 'overwhelmed' than empowered by the experience of testing. One of the key features of the MONITOR Trial is that we are focusing on HRQOL and other patient-centered aspects of SMBG and by the nature of the study design we are examining the impact of this self-care activity within the context of patient's having actionable knowledge and improved opportunities for provider/patient collaboration.

While researchers and medical organizations debate the overall issue of the value of SMBG testing in NIT $\mathrm{DM}$, patients face this choice daily and without adequate information as to its clinical or psychological outcomes. For some patients, their decision on testing will mirror that of their care provider. Yet more and more, patients play an active role in managing their own health. To some degree this is a necessary trend, because providers simply do not have sufficient time to provide intensive ongoing and comprehensive education and decisionmaking around diabetes self-management. Then too, even providers are looking for additional guidance on this question, including how to present options to patients and incorporate test results into care [7]. The fact that patients are taking a greater role in their health care is generally positive, because those who do so also improve their outcomes.

In order to make informed patient choices, patients and their providers need accurate, generalizable and meaningful information about the merits or demerits of SMBG testing. Because the existing research, though relatively extensive, has not yet met this important need, more research must be done on this issue. The Consensus
Report of the Diabetes Technology Society provides a list of recommendations for future research relating to SMBG. In regard to the intervention itself, they recommend that it a) is linked to a structured program designed to facilitate behavior change, b) has A1c as a primary endpoint but include patient-centered endpoints, c) include encouragement and support, preferably in the form of personalized, automated feedback to patients in real time, d) takes advantage of telemedicine opportunities, and d) incorporate best practices guidelines and standards for physicians [4, 15]. Many of these recommendations overlap with others' [32]. In addition to these critical features, future research should be designed with a pragmatic eye, adhering as closely as possible to the real world setting in which SMBG would be carried out by patients and utilized by patients and health care providers collaboratively. To date, no large-scale, pragmatic RCT has evaluated the impact of SMBG testing in patients with non-insulin treated T2DM in which a multi-dimensional approach to SMBG value management has occurred. This is the gap in knowledge that the MONITOR Trial will fill.

While SMBG may or not be worthwhile, effective SMBG, if it exists for non-insulin treated T2DM, appears to require that it be embedded within the context of patient education around the use and interpretation of glucose readings, provider awareness of the results of repeated testing, and collaborative use of this information at medical visits [33]. We would also argue for providing treatment algorithms to providers that are based on standard and accepted guidelines (such as the ADA guidelines) linked to SMBG report results [34]. This step facilitates the physician's use of glucometer result reports and can be used by health care providers during clinic visits to better illustrate their concerns when talking to patients. Finally, we feel it is important to evaluate objectively and in a real world setting the possible additional benefits of personalized feedback for patients in the form of messages delivered via the glucometer based on patients' current and recent SMBG patterns. By pointing out troubling patterns and rewarding results that are at goal, this aspect of the approach we are calling 'enhanced feedback' is akin to 'mini consultations' with a provider between routine clinic visits, which are generally 3-6 months apart.

\section{Abbreviations}

HPLC: Certified high performance liquid chromotography; ITT: Intention to treat; NGSP: National Glycohemoglobin Standardization Program; NIT DM: Non-insulin treated diabetes mellitus; SMBG: Self-monitoring of blood glucose; T2DM: Type 2 diabetes mellitus

Acknowledgements

None.

Funding

Research reported in this article was funded through a Patient -Centered Outcomes Research Institute (PCORI), 1828 L St., NW, Suite 900 Washington, 
DC 20036, Award \#CE-12-11-4980. The views presented in this article are solely the responsibility of the author(s) and do not necessarily represent the views of the Patient Centered Outcomes Research Institute (PCORI), its Board of Governors or Methodology Committee.

The project described also received supplementary support by the National Center for Advancing Translational Sciences (NCATS), National Institutes of Health, through Grant Award Number UL1TR001111. The content is solely the responsibility of the authors and does not necessarily represent the official views of the $\mathrm{NIH}$.

\section{Availability of data and materials}

Deidentified datasets used and/or analysed during the current study available from the corresponding author on reasonable request.

\section{Authors' contributions}

$L A Y, K E D, J B B$, and $M W$ were responsible for the study conception and design. $L A Y, K E D, J B B, M W, M V, J R, K G, T G$ and $M M$ were responsible for generating the patient messages and the treatment algorithm. JR, KG and TG were responsible for data collection. LAY, KED, JBB, MW, MV, JR, KG, TG, $A R$ and MM commented on the manuscript. LY was responsible for drafting the manuscript. All authors read and approved the final manuscript.

\section{Authors' information}

No additional information

\section{Competing interests}

L.A.Y. receives research Support (Paid to the University of North Carolina) from:

Boehringer-Ingelheim; Eli Lilly and Company; Gl Dynamics; Halozyme Therapeutics; Johnson and Johnson; Lexicon Pharmaceuticals, Inc:; Medtronic; Novo Nordisk, Inc.; Orexigen Therapeutics, Inc.; PhaseBio; Sanofi; Takeda Pharmaceuticals U.S.A., Inc.; ToleRx, Scion Neurostim, BMS J.B.B. is a consultant for and a shareholder of PhaseBio Pharmaceuticals and is a consultant/investigator for Andromeda, AstraZeneca, Boehringer Ingelheim, Bristol-Myers Squibb (BMS), Elcelyx Therapeutics, Eli Lilly and Company, Gl Dynamics, GlaxoSmithKline, Halozyme Therapeutics, F. Hoffmann-La Roche, Intarcia Therapeutics, Johnson \& Johnson, Lexicon, LipoScience, Medtronic, Merck, Metabolon, Metavention, Novo Nordisk A/S, Orexigen Therapeutics, Osiris Therapeutics, Pfizer, PhaseBio Pharmaceuticals, Quest Diagnostics, Rhythm Pharmaceuticals, Sanofi, Spherix, Takeda, ToleRx, and TransTech Pharma.

L.A.Y, J.B.B. and K.E.D report that UNC has licensed its interest in copyright works to Telcare of a glucose messaging and treatment algorithm for the purposes of commercialization.

\section{Consent for publication}

Not applicable.

\section{Ethics approval and consent to participate}

This project was approved by the University of North Carolina at Chapel Hil Institutional Review Board. Written informed consent was obtained from all participants.

\section{Publisher's Note}

Springer Nature remains neutral with regard to jurisdictional claims in published maps and institutional affiliations.

\section{Author details}

${ }^{1}$ Division of Endocrinology, Department of General Internal Medicine, School of Medicine, University of North Carolina at Chapel Hill, 8025 Burnett Womack Building, Campus Box \# 7172 UNC-CH, Chapel Hill, NC 27599-7170 USA. ${ }^{2}$ Cecil G. Sheps Center for Health Services Research, University of North Carolina at Chapel Hill, Chapel Hill, NC, USA. ${ }^{3}$ UNC Gillings School of Global Public Health, Chapel Hill, USA. ${ }^{4}$ Department of Family Medicine, School of Medicine, University of North Carolina at Chapel Hill, Chapel Hill, NC, USA. ${ }^{5}$ Center for Health Promotion and Disease Prevention, UNC Chapel Hill, Chapel Hill, USA. ${ }^{6}$ North Carolina Division of Public Health, Chapel Hill, USA.
Received: 7 June 2016 Accepted: 30 March 2017

Published online: 25 May 2017

\section{References}

1. Malanda UL, Bot SD, Nijpels G. Self-monitoring of blood glucose in noninsulin-using type 2 diabetic patients: it is time to face the evidence. Diabetes Care. 2013:36:176-8.

2. Standards of medical care in diabetes-2014. Diabetes Care. 2014;37 Suppl 1:S14-80

3. Allemann S, Houriet C, Diem P, Stettler C. Self-monitoring of blood glucose in non-insulin treated patients with type 2 diabetes: a systematic review and meta-analysis. Curr Med Res Opin. 2009;25:2903-13.

4. Clar C, Barnard K, Cummins E, Royle P, Waugh N, Aberdeen Health Technology Assessment G. Self-monitoring of blood glucose in type 2 diabetes: systematic review. Health Technol Assess (Winchester, England). 2010;14:1-140.

5. Farmer AJ, Perera R, Ward A, Heneghan C, Oke J, Barnett AH, Davidson MB, Guerci B, Coates V, Schwedes U, O'Malley S. Meta-analysis of individual patient data in randomised trials of self monitoring of blood glucose in people with non-insulin treated type 2 diabetes. Br Med J. 2012;344:e486-6.

6. Malanda UL, Welschen LM, Riphagen II, Dekker JM, Nijpels G, Bot SD. Selfmonitoring of blood glucose in patients with type 2 diabetes mellitus who are not using insulin. Cochrane Database Syst Rev (Online) 2012;1:CD005060.

7. Poolsup N, Suksomboon N, Rattanasookchit S. Meta-analysis of the benefits of self-monitoring of blood glucose on glycemic control in type 2 diabetes patients: an update. Diabetes Technol Ther. 2009;11:775-84.

8. Towfigh A, Ronnanova M, Weinreb JE, Munjas B, Suttorp MJ, Zhou A, Shekelle PG. Self-monitoring of blood glucose levels in patients with type 2 diabetes mellitus not taking insulin: A meta-analysis. Am J Managed Care. 2008;14:468-73.

9. Vijan S. Type 2 diabetes. Ann Intern Med. 2010;152:ITC31-15. quiz ITC316.

10. Istepanian RS, Zitouni K, Harry D, Moutosammy N, Sungoor A, Tang B, Earle KA. Evaluation of a mobile phone telemonitoring system for glycaemic control in patients with diabetes. J Telemed Telecare. 2009:15:125-8.

11. Bonomo K, De Salve A, Fiora E, Mularoni E, Massucco P, Poy P, Pomero A, Cavalot F, Anfossi G, Trovati M. Evaluation of a simple policy for pre- and post-prandial blood glucose self-monitoring in people with type 2 diabetes not on insulin. Diabetes Res Clin Pract. 2010;87:246-51.

12. Duran A, Martin P, Runkle I, Perez N, Abad R, Fernandez M, Del Valle L, Sanz MF, Calle-Pascual AL. Benefits of self-monitoring blood glucose in the management of new-onset Type 2 diabetes mellitus: the St Carlos Study, a prospective randomized clinic-based interventional study with parallel groups. J Diabetes. 2010;2:203-11.

13. Polonsky WH, Fisher L, Schikman CH, Hinnen DA, Parkin CG, Jelsovsky Z, Petersen B, Schweitzer M, Wagner RS. Structured self-monitoring of blood glucose significantly reduces A1C levels in poorly controlled, noninsulintreated type 2 diabetes: results from the Structured Testing Program study. Diabetes Care. 2011;34:262-7

14. Franciosi M, Lucisano G, Pellegrini F, Cantarello A, Consoli A, Cucco L, Ghidelli R, Sartore G, Sciangula L, Nicolucci A, Group RS. ROSES: role of selfmonitoring of blood glucose and intensive education in patients with Type 2 diabetes not receiving insulin. A pilot randomized clinical trial. Diabetic Med. 2011:28:789-96.

15. Klonoff DC, Blonde L, Cembrowski G, Chacra AR, Charpentier G, Colagiuri S, Dailey G, Gabbay RA, Heinemann L, Kerr D, et al. Consensus report: the current role of self-monitoring of blood glucose in non-insulin-treated type 2 diabetes. J Diabetes Sci Technol. 2011;5:1529-48.

16. Ware JE, Kosinski M, Bjorner JB, Turner-Bowker DM, Gandek B, Maruish ME. User manual for the SF-36V2 health survey. Lincoln: QualityMetric Inc; 2007.

17. Quality of life in type 2 diabetic patients is affected by complications but not by intensive policies to improve blood glucose or blood pressure control (UKPDS 37). U.K. Prospective Diabetes Study Group. Diabetes Care. 1999;22:1125-1136

18. Anderson RT, Narayan KM, Feeney P, Goff Jr D, Ali MK, Simmons DL, SperlHillen JA, Bigger T, Cuddihy R, O'Conner PJ, et al. Effect of intensive glycemic lowering on health-related quality of life in type 2 diabetes: ACCORD trial. Diabetes Care. 2011;34:807-12.

19. Edelman D, Olsen MK, Dudley TK, Harris AC, Oddone EZ. Impact of diabetes screening on quality of life. Diabetes Care. 2002;25:1022-6. 
20. Ware Jr JE, Sherbourne CD. The MOS 36-item short-form health survey (SF36). I. Conceptual framework and item selection. Med Care. 1992;30:473-83.

21. Snoek FJ, Pouwer F, Welch GW, Polonsky WH. Diabetes-related emotional distress in Dutch and U.S. diabetic patients: cross-cultural validity of the problem areas in diabetes scale. Diabetes Care. 2000;23:1305-9.

22. Welch $\mathrm{G}$, Weinger $\mathrm{K}$, Anderson B, Polonsky WH. Responsiveness of the Problem Areas In Diabetes (PAID) questionnaire. Diabetic Med. 2003;20:69-72.

23. Welch GW, Jacobson AM, Polonsky WH. The problem areas in diabetes scale. An evaluation of its clinical utility. Diabetes Care. 1997;20:760-6.

24. Herschbach P, Duran G, Waadt S, Zettler A, Amm C, Marten-Mittag B. Psychometric properties of the Questionnaire on Stress in Patients with Diabetes-Revised (QSD-R). Health Psychol. 1997;16:171-4.

25. Sacco WP, Bykowski CA, Mayhew LL. Pain and functional impairment as mediators of the link between medical symptoms and depression in type 2 diabetes. Int J Behav Med. 2013;20(1):22-29.

26. Van der Does FE, De Neeling JN, Snoek FJ, Kostense PJ, Grootenhuis PA, Bouter LM, Heine RJ. Symptoms and well-being in relation to glycemic control in type II diabetes. Diabetes Care. 1996;19:204-10.

27. Parchman ML, Flannagan D, Ferrer RL, Matamoras M. Communication competence, self-care behaviors and glucose control in patients with type 2 diabetes. Patient Educ Couns. 2009:77:55-9.

28. Schmidt WE, Christiansen JS, Hammer M, Zychma MJ, Buse JB. Patientreported outcomes are superior in patients with Type 2 diabetes treated with liraglutide as compared with exenatide, when added to metformin, sulphonylurea or both: results from a randomized, open-label study. Diabet Med. 2011;28:715-23.

29. Anderson RM, Fitzgerald JT, Gruppen LD, Funnell MM, Oh MS. The diabetes empowerment scale-short form (DES-SF). Diabetes Care. 2003;26:1641-2.

30. Dunnett CW, Tamhane AC. Step-up multiple testing of parameters with unequally correlated estimates. Biometrics. 1995;51:217-27.

31. O'Kane MJ, Bunting B, Copeland M, Coates VE. ESMON study group Efficacy of self monitoring of blood glucose in patients with newly diagnosed type 2 diabetes (ESMON study): randomised controlled trial. BMJ. 2008;336:1174-1177.

32. Farmer AJ, Wade AN, French DP, Simon J, Yudkin P, Gray A, et al. Blood glucose self-monitoring in type 2 diabetes: A randomised controlled trial. Health Technol Assess. 2009:13(15):1-50

33. Benhalima K, Mathieu C. The role of blood glucose monitoring in noninsulin treated type 2 diabetes: What is the evidence? Prim Care Diabetes. 2012;6(3):179-85.

34. Inzucchi SE, Bergenstal RM, Buse JB, Diamant M, Ferrannini E, Nauck M, et al. Management of hyperglycemia in type 2 diabetes: A patient-centered approach: Position statement of the american diabetes association (ADA) and the european association for the study of diabetes (EASD). Diabetes Care. 2012;35(6):1364-79

\section{Submit your next manuscript to BioMed Central and we will help you at every step:}

- We accept pre-submission inquiries

- Our selector tool helps you to find the most relevant journal

- We provide round the clock customer support

- Convenient online submission

- Thorough peer review

- Inclusion in PubMed and all major indexing services

- Maximum visibility for your research

Submit your manuscript at www.biomedcentral.com/submit 